\title{
Le Nouvel An chinois à Paris : théâtre d'économies ethniques
}

\section{Estelle Auguin}

\section{Q OpenEdition}

1 Journals

Édition électronique

URL : https://journals.openedition.org/remi/2021

DOI : 10.4000/remi.2021

ISSN : $1777-5418$

Éditeur

Université de Poitiers

\section{Édition imprimée}

Date de publication : 1 décembre 2004

Pagination : 75-87

ISBN : 2-911627-38-5

ISSN : 0765-0752

\section{Référence électronique}

Estelle Auguin, «Le Nouvel An chinois à Paris : théâtre d'économies ethniques », Revue européenne des migrations internationales [En ligne], vol. 20 - $n^{\circ} 3$ | 2004, mis en ligne le 25 septembre 2008, consulté le 14 avril 2022. URL : http://journals.openedition.org/remi/2021 ; DOI : https://doi.org/10.4000/remi. 2021

Ce document a été généré automatiquement le 14 avril 2022.

(c) Université de Poitiers 


\title{
Le Nouvel An chinois à Paris : théâtre d'économies ethniques
}

\author{
Estelle Auguin
}

1 La Tour Eiffel éclairée de rouge pendant la première semaine du calendrier lunaire est une image forte qui symbolise une amitié franco-chinoise et place dans le même temps la communauté chinoise sous les projecteurs médiatiques ${ }^{1}$. En effet, le défilé du Nouvel An chinois qui s'est déroulé, à l'occasion de l'année de la Chine en France ${ }^{2}$, sur l'avenue des Champs-Élysées en janvier 2004, donne à voir la communauté chinoise de France à la société française. Si les fêtes traditionnelles du calendrier chinois gardent une certaine prégnance au sein de l'existence des Chinois de la diaspora, le Nouvel An lunaire est au centre de la structuration temporelle de la communauté chinoise. Il revêt des enjeux divers permettant aux Chinois d'affirmer leur singularité. Dans le même temps, les processus identitaires s'actualisent dans un contexte particulier et relèvent de stratégies cultuelles, commerciales et politiques (Agier, $2000: 226$ ). Le Nouvel An chinois est un lieu privilégié d'analyse de la communauté chinoise dans la société française dans la mesure où elle se met en scène en s'adressant tant à un public intracommunautaire qu'à l'extérieur de la communauté.

2 Avant de comprendre l'importance de cette fête pour les Chinois de Paris, il convient de définir le terme de communauté chinoise. L'extrême hétérogénéité de la population chinoise de la capitale rend difficile l'emploi du terme de communauté. Un très grand nombre de Chinois de France est arrivé au milieu des années soixante-dix, fuyant les dictatures communistes que s'installaient dans les anciens pays d'Indochine et les massacres qui s'en suivirent, pays où cette population vivait depuis plusieurs générations parfois. C'est pourquoi il serait plus juste de parler d'Asiatiques plutôt que de Chinois. Toutefois, la population étudiée ici se réclame d'une origine chinoise. Bien que la famille de certains d'entre eux ait quitté la Chine depuis près d'une centaine d'années, ils se sentent appartenir à une culture chinoise, culture dont la langue écrite permet le "dépassement» (Trolliet, 2000: 85) de la diversité géo-dialectale et de l'éclatement diasporique. Cette diversité géo-dialectale à laquelle s'ajoute en France une grande hétérogénéité sociale peut conduire à parler de plusieurs communautés. 
Cela étant, dans cette étude, le terme de communauté chinoise est utilisé pour considérer la population chinoise et d'origine chinoise en opposition à la société autochtone.

3 Les fêtes de fin d'année constituent « un temps fort de la vie culturelle, de la consommation et de l'échange » (Raulin, 2000 : 99). Pour les Chinois, cette fête, appelée aussi fête du Printemps (chunjie) est l'une des plus importantes de leur calendrier. En Chine, elle est l'occasion d'un mois de vacances scolaires et de trois jours fériés. Elle a lieu durant la deuxième lune qui suit le solstice d'hiver, soit entre la fin janvier et le début de février. Ces dernières années, les festivités publiques du Nouvel An chinois sont devenues un rendez-vous important parmi les fêtes parisiennes. Des dizaines de milliers de personnes d'horizons divers viennent assister aux défilés dans la capitale. Pour les Chinois de Paris, que cela soit au sein des différentes associations, en participant au défilé ou bien en étant simples spectateurs, ils sont mis sous les feux des projecteurs durant ces quelques jours. D'une communauté d'ordinaire discrète, elle se transforme en l'acteur principal d'un théâtre prisé. Théâtre dont la mise en scène donne un cadre particulier pour l'analyse de la place de ce groupe de culture minoritaire au sein de la société française. Les acteurs principaux de cette fête sont moins les personnes d'origine asiatique que les associations chinoises qui mettent en avant leur identité culturelle.

4 Cependant, l'essentiel du Nouvel An chinois public ne réside pas dans la mise en scène de l'identité culturelle. Ces festivités sont, de fait, le lieu d'enjeux bien plus fondamentaux pour la communauté chinoise. Dès lors, cette dernière n'est plus la vedette exclusive de ce scénario: les leaders économiques, les autorités politiques françaises et chinoises jouent un rôle de premier plan dans ce jeu inter-ethnique. Les enjeux se situent à plusieurs niveaux: d'une part, au niveau intra-communautaire, d'autre part, sur le plan économique et commercial, enfin au niveau, politique.

5 Si cet article repose sur un matériau collecté lors du Nouvel An chinois 2003, ses conclusions restent pertinentes et trouvent même un regain d'actualité au regard du déroulement des festivités en 2004. Celles-ci ont un caractère exceptionnel en cette année de la Chine en France. À cette occasion, les associations chinoises de la capitale ainsi que la municipalité de Pékin sont conviées à participer à un gigantesque défilé sur les Champs-Élysées, organisé par la Mairie de Paris pour fêter l'entrée dans l'année du singe.

\section{Lieu d'une gestion de l'ethnicité}

6 Le Nouvel An lunaire est le lieu de la gestion de l'ethnicité des Asiatiques de Paris. L'événement autour duquel l'identité est mise en scène ; une mise en scène destinée à l'« entre soi », aussi bien qu'à la société autochtone.

7 Dès lors, le premier niveau des enjeux revêtus par le Nouvel An chinois se situe au sein de la communauté elle-même. La fête du Nouvel An est instrumentalisée dans le but de réinventer une identité à travers laquelle le groupe va se rassembler autour d'un sentiment d'appartenance. Par identité, il faut entendre le " processus de construction de sens » qui s'élabore à partir de matériaux empruntés à l'histoire, la géographie, « la mémoire collective » et "aux fantasmes personnels» se redéfinissant en fonction de "déterminations sociales » et de "projets culturels » dans un contexte singulier (Castells, 1999: 17-18). Au cours des nombreuses migrations, une même origine 
commune " réelle ou supposée » (Ma Mung, $2000: 9$ ) se construit au contact de cultures et d'individus différents, tout en gardant un lien de solidarité avec le reste de la diaspora. Il existe "une approche chinoise des faits humains" qui s'exprime en particulier dans la conception de la famille et des relations communautaires (Trolliet, 2000 : 85). Les Chinois appartiennent à une diaspora dans la mesure où ils constituent un réseau complexe de connaissances, de liens et d'échanges entre les différentes communautés chinoises du monde. Ce réseau ne peut exister qu'avec une conscience identitaire qui pousse les individus à croire qu'ils appartiennent à une même culture, ce qui les conduit à collaborer et s'entraider. Le Nouvel An lunaire, fêté dans le monde entier, donne à la communauté une visibilité de son réseau de connexion, lui permettant de se penser (et d'être pensée) en réseau diasporique.

Dans cette construction et gestion de l'identité, le Nouvel An chinois possède une place privilégiée dans la mesure où, malgré l'érosion des fêtes traditionnelles chinoises chez les jeunes générations, le Nouvel An lunaire garde une prégnance dans la communauté.

9 En premier lieu, l'identité se construit grâce, notamment, à des activités organisées au sein d'associations, mobilisant des éléments de culture chinoise qui sont sélectionnés (consciemment ou non) et conservés. Les associations organisent des activités qui rappellent à leurs membres leurs origines culturelles: cours de chinois pour les enfants, opéra chinois, ping-pong, kung-fu, etc. Toute l'année, elles y préparent les fêtes et les spectacles présentés à l'occasion du Nouvel An lunaire. L'intérêt des membres à s'investir dans les activités proposées est la participation non pas à une activité, sportive par exemple - les activités sportives ne nécessitant pas un investissement important - mais plutôt à une activité ethno-culturelle qui mobilise le temps d'une fête les membres de la communauté autour d'un enjeu culturel.

Durant les rassemblements associatifs qui marquent la fête du Printemps, des spectacles organisés mettent en scène les personnes d'origine chinoise et tout particulièrement les jeunes leur permettant de s'exprimer dans leur langue d'origine. Ils chantent en mandarin leur fierté d'être Chinois et proclament une identité commune: "Nous venons tous d'un pays qui se nomme la Chine ». Décors et costumes évoquent des éléments de la culture chinoise. Tout cela donne aux enfants, aux jeunes ainsi qu'aux plus âgés la possibilité d'apprécier l'importance d'une communauté réunie autour d'une identité. L'identité est ritualisée et permet d'observer «la culture “en train de se faire" » (Agier, $2000: 229$ ).

11 Le passage à la nouvelle année lunaire est également l'occasion pour les membres de la communauté de se réunir pour la veillée dans les associations et notamment celles qui abritent un temple. La communauté, réunie dans ce moment de la vie religieuse, se cristallise le temps d'une soirée, le temps d'une fête. Elle prend conscience de son existence. Dans une moindre mesure que la vie collective hivernale des Eskimo étudiée par Mauss, ce moment du calendrier crée pour la communauté chinoise «le sentiment que la communauté a d'elle-même, [...] son unité ». (Mauss, 1999 : 445).

12 En second lieu, le Nouvel An chinois devient un instrument culturel pour les groupes constituant la communauté chinoise afin d'affirmer une place particulière dans la société française. En effet, une culture singulière n'engendre pas obligatoirement une "identité différenciée », cette dernière est produite par des procédures de distinction développées par les individus en relation (Cuche, 2001 : 87). Ces procédures relèvent de stratégies de groupes qui sont d'autant plus visibles quand cette différentiation est interne à la communauté. En ce sens, les particularismes qui, entre deux cultures aussi 
dissemblables que les cultures française et chinoise, peuvent être communément imputés à la différence culturelle, ne peuvent plus cacher leur jeu quand ils sont internes à la communauté chinoise. Un particularisme géo-dialectal est visible durant les festivités publiques et communes du Nouvel An telles que le défilé. La plupart des associations qui y participent sont géo-dialectales et regroupent de riches commerçants. Elles relèvent de stratégies de solidarités financières qui prennent leur base sur la croyance en une origine commune ${ }^{3}$. La plupart des associations sont regroupées sur des bases économiques, ce qui conduit à des rapports de forces économiques internes à la communauté. Le défilé du Nouvel An chinois dans le XIII ${ }^{\mathrm{e}}$ arrondissement met en évidence ces différences et les stratégies de pouvoir déployées. À ce titre, la place de chaque association dans le parcours du défilé donne à voir le poids de chacune d'elles dans la hiérarchie d'influence du paysage associatif chinois.

Ces stratégies de pouvoir sont particulièrement visibles dans le XIII ${ }^{e}$ arrondissement où la plupart des associations sont dirigées par des Teochew, ceux-ci étant regroupés en fonction de la région d'Indochine qu'ils ont fuie dans les années soixante-dix. On y voit, à cet égard, une lutte entre « les frères ennemis » que sont $\mathrm{M}$. Rattanavan de la société Tang Frères et M. Trinh Huy de la société Paris Store. Cette hostilité interne aurait pu aboutir, en 2001, à la constitution de deux défilés concurrents dans le quartier si les autorités préfectorales ne l'avaient pas empêchée. En 2003, Tang frères étaient le financier du défilé dans le quartier chinois de la capitale. En 2004, alors que Paris Store aurait été l'organisateur du défilé s'il avait eu lieu dans le Triangle de Choisy, le cortège de l'association financée par le supermarché (ARFOI), défilait en seconde position sur les Champs-Élysées, juste derrière le cortège de la municipalité de Pékin. Quant à son concurrent direct (le CICOC financé par Tang frères), il était relégué en dernière position «pour des choix artistiques » assure une des responsables de l'organisation de WM Evénements, l'entreprise chargée de l'organisation du défilé par la mairie de Paris, en ajoutant que «la première ou la dernière place sont toutes deux des places d'honneur dans la culture chinoise ».

Ce particularisme géo-dialectal revendiqué dévoile moins une volonté de différentiation culturelle qu'une exacerbation de divergences infimes pour prendre le pouvoir économique et politique au sein de la communauté. L'identité se fonde sur des stratégies entre les groupes sociaux et devient «le support des luttes» (Cuche, 2001 : 94) économiques et politiques.

In fine, le milieu urbain, en multipliant les contacts d'individus renforce « les processus identitaires" (Agier, 2000: 226) et donne un cadre privilégié à l'expression de l'ethnicité. Cette dernière devient un enjeu fondamental de la fête du Nouvel An pour la communauté asiatique qui revendique une culture ancestrale.

\section{Enjeux économiques et reconnaissance politique}

En plus d'un aspect culturel, le Nouvel An chinois revêt des enjeux économiques et commerciaux. Les commerces chinois et les entreprises françaises, s'emparent de cette fête ethnique pour mettre en place des stratégies de vente et s'emparer d'un exotisme vendeur. Les Asiatiques de France sont essentiellement des commerçants qui ont su utiliser le créneau de l'exotisme pour développer une économie ethnique, une économie au sens de " phénomènes concernant la production, la distribution et la consommation des biens » (Rey, $2000: 1$ 173). 
17 Les communautés chinoises à travers le monde possèdent de nombreuses ressemblances entre elles. On remarque notamment qu'elles s'organisent autour d'un pôle entreprenarial. La puissance financière de cette population lui permet, grâce à un fort réseau de solidarité, de former une communauté marchande importante. La dimension identitaire de cette organisation économique est prépondérante. Elle revêt deux aspects. Le premier est la vente de produits ethniques. Les Chinois ouvrent, le plus souvent, des commerces asiatiques comme des restaurants, des supermarchés, etc. Le commerce ethnique relève d'une " négociation des identités ». En effet, le commerçant chinois, dans l'échange marchand, ne recherche pas uniquement le profit mais également «la reconnaissance du produit comme production de son identité ». (Ma Mung, 2000 : 139). Le second aspect est la formation d'un marché ethnique du travail avec notamment l'emploi de la part des commerces d'une main-d'œuvre provenant en grande majorité de la communauté. Dès lors, l'importance identitaire de la fête du Printemps prend toute son ampleur pour cette communauté marchande dont le commerce repose sur un caractère ethnique installé sur un territoire identifié. Ces entreprises s'approprient l'espace urbain le temps de la fête du Nouvel An chinois donnant à cette fête publique un caractère publicitaire.

18 Premièrement, les commerces ethniques profitent de la fête pour attirer du monde dans les quartiers chinois. Le marquage ethnique du territoire, nécessaire à «l'attraction urbaine» (Raulin, 1990b : 25), devient un théâtre de représentation pour les Chinois au moment de leur Nouvel An. À cet égard, la danse du lion, devenue aujourd'hui emblématique de la fête du Printemps, passe chaque année le jour de la nouvelle année lunaire dans les quartiers chinois de la capitale. D'ailleurs, la centralisation des festivités sur les Champs-Élysées en 2004 n'a pas empêché son organisation devant les commerces ethniques le matin du premier jour du calendrier lunaire. Cette tradition s'est développée sur un terreau commercial. Les lions chassent les mauvais esprits loin des commerces et séduisent de très nombreux spectateurs curieux, attirés par le bruit des pétards et par la foule. La danse du lion compte aussi une dimension sociale dans la mesure où elle est pour les commerçants un signe de reconnaissance qui leur permet d'affirmer leur prestige. En effet, les enveloppes rouges offertes aux lions, contenant plusieurs dizaines d'euro, permettent de montrer la prospérité du commerce.

19 Cette tradition festive, en attirant du monde dans le quartier et devant les étalages des commerçants, revêt un caractère publicitaire. En ce sens, l'identité de ces groupes se négociant dans les transactions marchandes (Ma Mung, 2000: 139), la fête du Printemps est l'occasion pour ces commerces ethniques de faire de la publicité. Dans le $\mathrm{XIII}^{\mathrm{e}}$ arrondissement, le défilé 2003 attire quelques cent mille personnes. Nombres de magasins restent ouverts pour la journée et profitent du désir d'exotisme des badauds fascinés par les couleurs de la fête. Beaucoup d'autochtones passent dans les boutiques chinoises à la fin du défilé pour s'acheter un souvenir ou un produit introuvable ailleurs. À partir de $11 \mathrm{~h} 30$ et durant toute l'après-midi, les restaurants asiatiques affichent complets notamment ceux qui donnent sur les avenues dans lesquelles se déroule le défilé, ce qui permet aux clients de constater la présence de la fête, sans pour autant en voir son déroulement, la foule leur cachant le cortège. Quant au défilé 2004, le prestige des Champs-Élysées, bien qu'étant une grande fierté pour la communauté, n'a pas empêché nombre de commerçants d'exprimer leur déception de la désertion du quartier pour un jour si fructueux pour leur commerce. 
20 Deuxièmement, les grandes enseignes de ventes de produits ethniques profitent également de la fête pour faire de la publicité. Ainsi, le supermarché Tang frères est le maître de la fête de l'année 2003. De plus, alors qu'aucune festivité n'est officiellement organisée en 2004, il marque de sa présence les avenues de Choisy et d'Ivry par des banderoles publicitaires sur les lampadaires. Deux enseignes lumineuses Tang Frères souhaitent également une bonne année à tous les passants et clients, l'une d'elle est installée au-dessus de l'avenue d'Ivry et l'autre à l'entrée du parking du supermarché. Tang frères est l'un des plus puissants groupes d'importateurs contrôlant l'ensemble du réseau d'importation de produits alimentaires asiatiques. (Huu Khoa, 1996: 66). Officiellement, c'est le $\mathrm{CICOC}^{4}$ qui est chargé pour l'entrée dans l'année de la chèvre en 2003 d'organiser la fête ainsi que de défiler sur les Champs-Élysées en 2004, les associations étant les seules invitées à le faire. Mais, c'est Tang frères qui couvre les frais. À ce titre, jamais le nom du CICOC n'est évoqué seul; sur toutes les affiches, brochures ou banderoles, la mention « avec le concours de Tang frères » accole celle " organisé par le CICOC ».

21 Troisièmement, le Nouvel An chinois n'est pas instrumentalisé seulement par les commerçants asiatiques profitant de la fête pour attirer des clients inhabituels, les autochtones ont depuis plusieurs années compris les possibilités commerciales offertes par ce filon exotique et festif. D'une part, on peut voir durant le défilé les habitués des fêtes publics : les vendeurs ambulants qui installent leur stand de bonbons, de pétards, de confettis dans la foule. Certains vendent des ballons gonflés à l'hélium. Les vendeurs ponctuels sont des Français ou des personnes d'origine maghrébine. D'autre part, les entreprises françaises semblent s'être emparées de cette fête ethnique pour proposer des produits à l'occasion du Nouvel An chinois. Ainsi, on remarque que certains magazines ${ }^{5}$ proposent l'horoscope chinois à leurs lecteurs; de plus, des enseignes de supermarchés proposent des produits asiatiques durant cette période de l'année, trouvant ici une occasion supplémentaire entre Noël et la Saint Valentin de faire de la publicité commerciale.

Finalement, cette économie stable et puissante donne à la communauté une image de réussite dans la société autochtone. En l'occurrence, la réussite économique leur permet d'affirmer, pendant la fête, leur "positionnement social» dans la société française (Agier, $2000: 197)$.

\section{Lieu d'une reconnaissance politique}

En prenant l'importance médiatique et commerciale qu'il connaît aujourd'hui, le Nouvel An chinois organisé dans l'espace public revêt un rôle politique central, politique au sens de gestion des intérêts et des objectifs de chacun, gestion des interdépendances sociales. C'est alors qu'interviennent les intérêts économiques qui sont fondamentaux dans la compréhension des rapports politiques entre les Chinois résidant en France et les autorités françaises. À ce titre, les fêtes permettent de «lire les stratégies pour se faire reconnaître » et de comprendre la façon dont les acteurs sociaux développent des liens relationnels dans une ville ou un quartier. (Hily et Meintel, 2000 : 7). D'un côté, les organisateurs du Nouvel An chinois utilisent leur culture pour affirmer voire pour revendiquer une place dans l'espace politique, économique et social français; de l'autre, les autorités politiques qu'elles soient 
françaises ou chinoises trouvent dans cette fête l'occasion de donner à cette communauté dynamique économiquement une reconnaissance sociale.

\section{Le rôle des élus parisiens} différents face aux communautés chinoises importantes qui résident dans leurs quartiers. D'un côté, la mairie du XIII earrondissement n'a ni un rôle moteur ni un rôle de financement dans les nombreuses festivités organisées pour le Nouvel An chinois. De fait, les rapports de force internes à la communauté obligent la mairie à intervenir dans l'organisation de la fête en jouant un rôle de médiation entre les différents clans de la communauté. Cependant, elle ne souhaite pas créer un défilé municipal et leur reconnaît un rôle fondamental pour la vie du quartier. Par ailleurs, le poids économique des Chinois et le dynamisme qu'ils ont créés dans l'arrondissement encouragent la mairie à s'intéresser à cette population; elle n'oublie pas non plus que ses interlocuteurs sont aussi des électeurs potentiels. De l'autre, dans le III ${ }^{e}$ arrondissement, c'est l'équipe municipale qui est ici à l'initiative des festivités. Aidée par les associations chinoises et franco-chinoises du quartier 6 , elle organise une semaine culturelle chinoise pour accompagner la communauté la plus importante numériquement du quartier dans leur fête emblématique. Cette organisation culturelle dévoile une main tendue des autorités françaises vers cette communauté étrangère pour faciliter son intégration à la vie locale. De plus, on peut remarquer que les activités proposées sont dirigées autant, voire davantage, vers la population autochtone que vers la communauté chinoise. C'est aussi dans un esprit politique au sens de valeurs idéologiques qu'une semaine culturelle chinoise est organisée. On peut souligner, par ailleurs, que la semaine chinoise entre dans une politique plus globale de la mairie d'arrondissement dans laquelle des événements culturels sont régulièrement organisés.

En second lieu, le maire de Paris, Bertrand Delanoë, est très attendu aux cérémonies du Nouvel An chinois dans lesquelles lui est réservée une place privilégiée. Il annonce le départ des cortèges des défilés en allumant des guirlandes de pétards, signe du commencement des festivités. Bien qu'il ne participe pas au défilé, il marque de sa présence la fête chinoise. En outre, il organise dans les locaux de l'Hôtel de ville une grande réception pour le Nouvel An chinois. Plus de trois mille personnes y sont conviées. Les cartons d'invitations sont distribués par l'intermédiaire des associations chinoises de toute la capitale. Ce jour-là, les présidents des associations chinoises, qui sont quasiment tous de riches commerçants de Paris, entrent par une porte réservée et sont invités à se placer, debout également, au premier rang, devant la tribune. Ils sont une trentaine, séparés du reste de la foule par une corde faisant barrière. C'est à eux que, de fait, le discours du maire est adressé. C'est un discours élogieux, immédiatement traduit en mandarin, destiné à une communauté asiatique «importante pour la ville de Paris». Il exhorte les Chinois à « être totalement [eux]mêmes » afin de «donner à Paris [leur] esprit d'entreprise, [leur] culture, et permettre à la capitale d'être différente ».

Revue européenne des migrations internationales, vol. 20 - n³| 2004 

communauté dont l'intégration économique sans cesse valorisée met au second plan une intégration sociale essentiellement intracommunautaire. De fait, durant toute la durée des festivités, les problèmes posés par les Chinois de France tels que le travail illégal, la clandestinité d'une partie de la population, la mono-activité des commerçants de certains quartiers, etc. sont occultés pour faire place aux aspects positifs de leur présence en France.

La place accordée au maire de Paris dans les cérémonies organisées à l'occasion de la fête du Printemps montre la volonté des Chinois d'obtenir une reconnaissance plus large que celle des seules instances politiques de leur quartier. L'accueil donné par le représentant politique de la capitale dans les locaux de la ville est à la mesure de leur poids économique dans le paysage parisien. M. Delanoë est non seulement conscient du dynamisme économique de la communauté chinoise mais, va également jusqu'à les inviter à «donner cet esprit d'entreprise» à la première ville française. La reconnaissance politique accordée à la communauté chinoise par les autorités politiques françaises met en valeur leur capacité d'intégration reposant sur «leur utilité sociale et économique $»^{7}$ (Ma Mung, $\left.2000: 153\right)$.

erte invitation à l'Hôtel de ville, une place toute particulière est réservée aux présidents d'associations séparés physiquement par une corde du reste de la foule. Cette hiérarchie officialisée dans les locaux mêmes de la République française marque la volonté politique d'une reconnaissance spécifique de l'élite dynamique chinoise essentiellement marchande. L'enjeu de part et d'autre est de taille car la reconnaissance du leadership de cette communauté n'est pas seulement symbolique. Pour la communauté chinoise, il peut également prendre l'aspect d'obtention de marchés, d'accords politiques pour éviter des problèmes urbains, etc. Pour les autorités françaises, les Chinois de France peuvent être une interface importante dans les relations diplomatiques entre la France et la Chine. Le marché chinois étant économiquement prometteur, mais cependant difficile à pénétrer pour des entreprises occidentales, les alliés français d'origine chinoise sont alors des atouts importants pour la France. À cet égard, il semble qu'une cérémonie ait également été organisée pour le Nouvel An chinois à l'Élysée. Jacques Chirac, longtemps maire de la capitale, a conservé de très bonne relation avec cette communauté qu'il sait entreprenante ; il a conscience qu'elle peut être un atout pour le pays dans ces relations avec le géant chinois. Ainsi, avec sa puissance démographique et économique de premier plan, la diaspora chinoise est au cœur des relations internationales.

\section{Le rôle des représentants chinois}

Les relations entre la Chine et sa diaspora constituent un problème historique complexe. Avec l'arrivée au pouvoir des communistes en 1949, la politique du pays visà-vis des Chinois de l'étranger est abordée sous «l'angle idéologico-politique » (Bourbeau, 2002: 63). Mais, à partir de 1978, Deng Xiaoping et les réformistes de la période post-maoïste bouleversent les relations avec la diaspora, en réorientant leur discours officiel sur de nouvelles bases économiques. La République populaire de Chine qui lance une grande réforme économique comprend alors que la diaspora chinoise est très puissante économiquement. La Chine fait alors appel aux Chinois d'outre-mer qui deviennent la majorité des investissements étrangers.

Revue européenne des migrations internationales, vol. 20 - n³ | 2004 
31 Dans cette optique, la place de l'ambassadeur Wu Jianmin dans les festivités du Nouvel An chinois en 2003 et la collaboration de la municipalité de Pékin pour le défilé sur les Champs-Élysées en 2004 sont les signes de cette attitude particulière que la Chine a établie avec sa diaspora depuis maintenant vingt ans. L'intérêt du gouvernement chinois, mais également celui des Chinois d'outre-mer, consiste en l'instauration entre les deux parties d'un lien fondé sur la recherche d'un bénéfice économique commun.

Premièrement, lors des festivités organisées pour l'entrée dans l'année de la chèvre en 2003, l'ambassadeur est présent dans les deux cérémonies du Nouvel An chinois célébrées par les communautés distinctes de la capitale. Dans le XIII ${ }^{\mathrm{e}}$ arrondissement, l'ambassadeur de Chine est invité par les associations chinoises, alors que ce sont les autorités municipales qui l'ont invité dans le $\mathrm{III}^{\mathrm{e}}$. La présence du représentant de la République Populaire de Chine dans les deux arrondissements permet de constater la prise de conscience par les autorités officielles chinoises de l'hétérogénéité de la diaspora. Cette prise en compte du gouvernement chinois l'a conduit à envisager un critère qui réunit dans un projet commun des générations très diversifiées de Chinois : l'économie (Bourbeau, 2002: 71-72). Pour les Chinois de France, la reconnaissance de l'ambassade est un honneur. En effet, il ne faut pas oublier qu'ils sont expatriés. La reconnaissance de leur réussite par leur patrie d'origine est importante. Réciproquement, l'État chinois a besoin de montrer à ses anciens compatriotes toute sa gratitude afin de garder des liens amicaux pour les affaires économiques.

Cela étant, le gouvernement chinois cherche aussi à entretenir avec les pays d'accueil des relations de bonne entente. Dans cette optique, la Chine souhaite privilégier ses relations bilatérales étatiques (Bourbeau, 2002: 83-84). Lors de ses discours aux cérémonies, l'ambassadeur parle des convergences de point de vue entre la France et la Chine sur la scène internationale. Pour lui, le partenariat sino-français est en phase ascendante sur trois piliers: politique, économique, et culturelle. Tout d'abord politique avec « la convergence de vision d'un monde multipolaire ». Le deuxième pilier de ce partenariat est économique avec la "multiplication des échanges économiques », notamment par les investissements français en Chine. C'est sans doute le point fondamental qui encourage un lien diplomatique cordial. En effet, que ce soit la République Populaire de Chine qui a besoin de capitaux pour son développement ou la France qui regarde avec envie l'immense marché chinois, les intérêts économiques permettent aux États la pacification de leurs relations ${ }^{8}$. Enfin, le troisième pilier est culturel. En effet, la France ouvrira un centre de la culture chinoise pour l'inauguration de l'année de la Chine en France, à la fin de l'année 2003. Réciproquement, un centre culturel français en Chine devrait ouvrir ses portes avec l'année de la France en Chine en 2004. Il souligne que c'est la première fois que la Chine fait cela avec un pays étranger. "Le monde du XXI ${ }^{\mathrm{e}}$ siècle a besoin du mélange des civilisations alors que le choc des civilisations serait terrible." Les festivités chinoises en France montrent justement que le dialogue est réalisable. Il est possible de «maintenir l'identité culturelle, la mondialisation ne signifie pas la perte de culture. » Il conclut en déclarant que « les Chinois sont toujours confiants dans l'avenir. »

Ce discours diplomatique de l'ambassadeur adressé aux autorités françaises dans un très bon français montre que même si la logique idéologique est dépassée dans les relations entre l'État chinois et la diaspora chinoise, l'État chinois continue d'utiliser les Chinois d'outre-mer pour atteindre «ses objectifs politico-stratégiques » (Bourbeau, 
2002 : 84). Les Chinois d'outre-mer servent de tremplin afin d'agrandir la sphère d'influence de la République Populaire de Chine.

Deuxièmement, à l'occasion de l'année de la Chine en France en 2004, des autorités chinoises de premier plan sont conviées à marquer de leur présence le Nouvel An chinois. Ainsi, le Président français profite de cette période festive pour recevoir son homologue chinois $\mathrm{Hu}$ Jintao en visite d'État afin de célébrer le quarantième ${ }^{9}$ anniversaire du rétablissement des relations diplomatiques entre la France et la République Populaire de Chine. De plus, le maire de Pékin est invité à défiler au côté du maire de Paris sur les Champs-Élysées et une délégation d'artistes pékinois ouvre le cortège. Ces participations officielles de représentants de l'État autoritaire chinois ne vont pas sans interroger notre tradition démocratique ${ }^{10}$. En effet, certaines associations n'ont pas le droit de participer à la fête. En ce sens, le Falungong, groupe de méditation fortement réprimé en République Populaire de Chine, est exclu du défilé pour « des raisons artistiques » selon l'entreprise organisatrice du défilé. On observe de fait, une pratique politique ambivalente: d'un côté le choix de la mairie de Paris est de privilégier les autorités légales chinoises en les accueillant officiellement lors des manifestations du défilé ; de l'autre, le premier magistrat de la capitale ne reconnaît pas les représentants associatifs défendant les droits de l'homme, dont la France se veut pourtant le héraut universel.

\section{Conclusion}

$\mathrm{Au}$ total, les festivités publiques du Nouvel An chinois dépassent largement le seul enjeu culturel pour une communauté expatriée en recherche d'identité. Elles sont le théâtre d'enjeux plus globaux pour une population enracinée dans le paysage français. Les acteurs de cette mise en scène sont des personnages publics tels que des élus de la capitale, l'ambassadeur de chine ou encore de riches commerçants-présidents d'associations. Ce sont également les nombreux membres des associations qui participent aux défilés en tant que participants ou spectateurs. Toutefois, il existe aussi un non-acteur qui est l'oublié de cette représentation de la communauté chinoise en France : une part non négligeable des membres de la communauté chinoise ne participe pas à ces festivités publiques. Il y a d'un côté, les familles d'immigration relativement récente, faiblement acculturées qui préfèrent fêter chez elles, comme elles le faisaient en Chine, ce moment familial. De l'autre, les immigrés des migrations très récentes, ceux des flux continuels qui ne cessent d'arriver en France et en Europe et qui sont, malgré une ouverture de la part de la mairie du troisième arrondissement, majoritairement absents. Clandestins, pauvres et bien souvent exploités, leur absence ne cache pas moins leur importance démographique grandissante et ne peut que nous interroger quant à leur avenir dans la société française. 


\section{BIBLIOGRAPHIE}

AGIER Michel (2000) Anthropologie du carnaval. La ville, la fête et l'Afrique à Bahia, Marseille, coédition : Éditions Parenthèses, Institut de Recherche pour le Développement.

BARTH Fredrik (1969) Les groupes ethniques et leurs frontières in Philippe Poutignat et Joceyne Streiff-Fenart (1999) Théories de l'ethnicité, Paris, PUF, [1995], pp. 203-249.

BERTHELEU Hélène (2000) Cohésion sociale, ethnicité et hiérarchies : fêtes et rituels lao en France, Revue Européenne des Migrations Internationales, 16 (2), pp. 153-170.

BOURBEAU Philippe (2002) La Chine et la diaspora chinoise, Paris, L’Harmattan, $150 \mathrm{p}$.

CASTELLS M., 1999, Le pouvoir de l'identité. L'ère de l'information, Paris, Fayard, (édition originale : 1997).

COSTA-LASCOUX Jacqueline et YU-SION Live (1995) Paris XIIIe, lumières d'Asie, Paris, Éditions Autrement, $167 \mathrm{p}$.

CUCHE Denys (2001) La notion de culture dans les sciences sociales, Paris, Éditions La Découverte, [1996], $123 \mathrm{p}$.

GUIHEUX G., 1996, La place des Chinois d'outre-mer en Chine, Politique étrangère, $\mathrm{n}^{\circ} 2$.

GUILLON Michelle et MA MUNG Emmanuel (Dir.) (1992), La diaspora chinoise en Occident, Revue Européenne des Migrations Internationales, 8 (3).

GUILLON Michelle et TABOADA LEONETTI Isabelle (1986) Le triangle de Choisy, un quartier chinois à Paris, Paris, L'Harmattan, $210 \mathrm{p}$.

HILY Marie-Antoinette et MEINTEL (Dir.) (2000) Fêtes et rituels dans la migration, Revue Européenne des Migrations Internationales, 16 (2).

HUU-KHOA Le (1996) L'immigration asiatique à Paris, Paris, CHEAM, $190 \mathrm{p}$.

MA MUNG Emmanuel (2000) La diaspora chinoise, géographie d'une migration, Paris, Ophrys, 176 p.

MAUSS Marcel (1999) Essai sur le don. Forme et raison de l'échange dans les sociétés archaïques in Sociologie et anthropologie, Paris, PUF, [1950 pour l'ouvrage ; 1923 pour l'article], pp. 143-279.

POUTIGNAT Philippe et STREIFF-FENART Jocelyne (1999) Théories de l'ethnicité, Paris, PUF, [1995], $270 \mathrm{p}$.

RAULIN Anne (2000) L'ethnique est quotidien, Paris, L'Harmattan.

RAULIN Anne (1991) Minorités intermédiaires et diasporas, Revue Européenne des Migrations Internationales, 7 (1), pp 163-170.

RAULIN Anne (1990a) Nouvel an asiatique et nouvel an occidental dans le quartier chinois de Paris, Dialogue, $\mathrm{n}^{\circ} 110$.

RAULIN Anne (1990b) Consommation et adaptation urbaine des minorités en région parisienne, Sociétés contemporaines, $\mathrm{n}^{\circ} 4$.

RAULIN Anne (1988) Espaces marchands et concentrations urbaines : la petite Asie à Paris, Cahiers internationaux de sociologie, volume 85.

REY Alain (Dir.) (2000) Le Robert dictionnaire historique de la langue française, Paris, Le Robert, [1992], 4294 p. (trois tomes). 
TROLLIET Pierre (2000) La diaspora chinoise, Paris, PUF, [1994], 128 p.

\section{NOTES}

1. Plusieurs dossiers sur les Chinois de France ont été publiés la semaine du 22 janvier 2004, jour du Nouvel An chinois. Citons, à titre d'exemple, le dossier de Libération le 21 janvier 2004.

2. L'année de la France en Chine se déroule de l'automne 2003 jusqu'à l'été 2004. elle est l'occasion de nombreuses activités culturelles chinoises ou sur la Chine telles que des expositions, des concerts, des opéras, etc. Elle sera succédée par une année de la France en Chine à partir de l'automne 2004.

3. Cette origine commune peut être régionale (les associations regroupent notamment les gens qui parlent le même dialecte) voire familiale (elles peuvent aussi regrouper les personnes de même nom ; l'origine familiale commune peut alors être seulement imaginée, le nombre restreint de noms de famille en Chine rendant les homonymes très fréquents).

4. Le Conseil pour l'intégration des communautés d'origine chinoise en France (CICOC) est une association fondée en 1988 financé par le magasin Tang frères.

5. Notons notamment Modes \& Travaux de janvier 2003 et 2004 ou Paris Match, qui fait un numéro spécial Chine tous les ans à cette époque.

6. Citons à titre d'exemple l'Association des Chinois résidant en France et l'association francochinoise Pierre Ducerf.

7. À ce propos, on peut souligner que le défilé du Nouvel An chinois a lieu l'année 2004 sur l'avenue la plus représentative de la nation française : les Champs-Élysées.

8. Ceci ne vaut évidemment pas uniquement pour les relations franco-chinoises; les intérêts économiques peuvent permettre la pacification des relations d'Etat, comme le montre Albert 0 . Hirschman dans Les passions et les intérêts (1997, Paris, PUF, première édition : 1980).

9. La France est l'un des premiers pays de l'Occident à reconnaître la République Populaire de Chine. Avant 1964 pour la France et jusqu'en 1971 pour l'ONU et la plupart des autres nations occidentales, la République de Chine de Chiang Kai-shek (Taiwan) représente la Chine. La reconnaissance de la RPC implique depuis cette époque la non-reconnaissance diplomatique de Taiwan.

10. Des intellectuels français et des associations réagissent vivement : on peut lire notamment Jean-Philippe Béja, sinologue, s'indigner de la présence aux festivités des autorités pékinoises, héritières du massacre de la place Tiananmen en 1989 (Le Monde du 21 janvier 2004) ou encore voir des manifestations pro-tibétaines en parallèles du défilé des Champs-Élysées.

\section{INDEX}

Mots-clés : Chinois, économie ethnique, fête, tradition 
AUTEUR

ESTELLE AUGUIN

Estelle AUGUIN prépare une thèse de socio-démographie à POPINTER, Université Paris 5 René

Descartes, 45, rue des Saints Pères 75006 Paris. www.shs.univ-paris5/popinter/ 